\title{
Causes of lower urinary tract disease in Czech cat population
}

\author{
Barbora Hřibová ${ }^{1}$, Václav Ceplecha ${ }^{1}$, Kristina Řeháková2 ${ }^{2}$ Pavel Proks ${ }^{1,4}$ \\ Vojtěch Gabriel $^{1}$, Ludmila Kohoutová ${ }^{3}$, Michal Crha ${ }^{1}$
}

University of Veterinary and Pharmaceutical Sciences Brno, Faculty of Veterinary Medicine, ${ }^{1}$ Small Animal Clinic, ${ }^{2}$ Small Animal Clinical Laboratory, ${ }^{3}$ Department of Infectious Diseases and Microbiology, ${ }^{4}$ CEITEC - Central European Institute of Technology, Brno, Czech Republic

Received May 30, 2019

Accepted October 29, 2019

\begin{abstract}
This study was done to investigate epidemiological data and to report causes of lower urinary tract disease in a population of cats presented at the Small Animal Clinic of the University of Veterinary and Pharmaceutical Sciences Brno. Cats presented with lower urinary tract disease signs that had undergone a thorough physical examination and urinalysis (dipstick, urine specific gravity, urine sediment and dipslide urine culture) were included in the study. Urine samples were collected only by cystocentesis or sterile catheterization. Bloodwork, abdominal ultrasound, and abdominal radiographs were performed in 118 $(66 \%), 170(96 \%)$ and $9(5 \%)$ patients, respectively. Cats that were treated with antibiotics or glucocorticoids during an episode of feline lower urinary tract disease (FLUTD) or during the foregoing month and which had undergone perineal urethrostomy or catheterization in private practice, were excluded. The study population consisted of 177 cats. Forty-one (23\%) cats were diagnosed with a urethral plug, 26 cats (14\%) with a urinary tract infection (UTI), 9 cats $(5 \%)$ with urolithiasis and 101 cats $(57 \%)$ with feline idiopathic cystitis (FIC). The cats diagnosed with UTI were significantly older than the cats with FIC, urethral plugs and urolithiasis. Urinary tract infection was diagnosed significantly more often in patients older than 10 years, and in female cats. The diagnosis of urethral plug was made significantly more often in males. Feline idiopathic cystitis and urethral plugs are the most common causes of FLUTD, and the causes are significantly age and sex-related.
\end{abstract}

FLUTD, UTI, LUTS, urethral plug, urolithiasis, feline

The term "Feline Lower Urinary Tract Disease" (FLUTD) summarizes a spectrum of clinical signs related to diseases of the urinary bladder and/or urethra. By aetiology, FLUTD covers multiple disorders such as urolithiasis, urinary tract infections (UTI), neoplasia, congenital or acquired morphological abnormalities, and feline idiopathic cystitis (FIC). Cats with FLUTD are often presented for stranguria, haematuria, periuria, pollakiuria, overgrooming of the genital area and inner thighs, and behavioral changes (i.e. aggression) (Gunn-Moore 2003). These lower urinary tract signs (LUTS) or their combinations are not specific for any particular disease as lower urinary tract reacts to all kinds of irritation in the same manner (Osborne et al. 1996).

Feline idiopathic cystitis is a diagnosis of exclusion (Forrester and Towell 2015). A diagnostic work-up including urinalysis (dipstick, urine sediment examination and urine culture), survey radiographs for identification of radiodense urethrolits/cystoliths and/or ultrasonographic examination for the identification of radiolucent uroliths or neoplastic changes should be performed to rule out other specific causes (Kruger et al. 2009). Many authors agree that FIC is the most common cause of LUTS (Kruger et al. 1991; Lekcharoensuk et al. 2001).

Significant differences regarding the prevalence of UTI in cats presenting for FLUTD were found. European studies (Netherlands, Switzerland, Norway, Germany and Poland) revealed a higher occurrence of FLUTD cases due to bacterial infection, ranging from $7.8 \%$

Address for correspondence:

MVDr. Barbora Hřibová

University of Veterinary and Pharmaceutical Sciences Brno

Palackého tř. 1946/1

Phone: + 420541562357

61242 Brno, Czech Republic 
to $22 \%$ (Kraijer et al. 2003; Gerber et al. 2005; Eggertsdottir et al. 2007; Dorsch et al. 2014; Lew-Kojrys et al. 2017) compared to studies from USA which had a prevalence up to $3 \%$ in cats less than 10 years of age (Kruger et al. 1991; Lekcharoensuk et al. 2001). Older female cats are generally at increased risk for the development of urinary tract infection (George 1996; Litster et al. 2009). The aging process and/or concurrent diseases, which are commonly seen in geriatricpatients, could contribute to reduction of natural urinary tract defense mechanisms against infection in older cats (George 1996). The occurrence of UTI is also increasing in cats with concurrent disorders such as chronic kidney disease, diabetes mellitus, or hyperthyroidism (Bailiff et al. 2006; Mayer-Roenne et al. 2007), after repeated catheterizations and perineal urethrostomy (Lekcharoensuk et al. 2001). Mayer-Roenne et al. (2007) found an association between decreasing urine specific gravity (USG) and increasing frequency of UTI in cats with diabetes mellitus. However, another study failed to prove this association (Bailiff et al. 2008).

Urethral plugs are believed to be the most common cause of urethral obstruction. In one study, urethral plugs were found to be the cause of obstruction in $55 \%$ of patients, with urolithiasis in $12 \%$, and the cause remaining unknown in $30 \%$ of cats (Kruger et al. 1991).

The aim of this retrospective study was to investigate epidemiological data and to report causes of lower urinary tract disease in a population of cats presented at the Small Animal Clinic, University of Veterinary and Pharmaceutical Sciences Brno (UVPS).

\section{Materials and Methods}

Medical records of cats which had been admitted to the Small Animal Clinic at UVPS from January 2013 to February 2019 with LUTS (pollakiuria, stranguria, haematuria, periuria, abnormal grooming of the genital area, vocalization on urination, straining to urinate) and diagnosed with FLUTD (obstructive or non-obstructive form) were reviewed and included in the study. Data retrieved from medical records included: breed, age, sex, neutering status, season of presentation (winter [December, January, February] spring [March, April, May] summer [June, July, August] autumn [September, October, November]), presence of urethral obstruction, urinalysis results, and final diagnosis. Cases were identified by searching medical records for "FLUTD", "FIC", "dysuria", "haematuria", "periuria" and "urethral obstruction". Cats that presented with their first episode were included along with cats with previous episodes of FLUTD. Animals that had been treated with antibiotics or glucocorticoids during the episode of FLUTD or during the foregoing month and that had undergone perineal urethrostomy or catheterization in private practice, were excluded. A thorough physical examination and dipslide urine culture were performed in all of the included cats. Bloodwork, abdominal ultrasound, and abdominal radiographs were performed in 118 $(66 \%), 170(96 \%)$ and $9(5 \%)$ of the patients, respectively.

The patients were divided into four groups (FIC, UTI, urethral plug, urolithiasis) based on defined diagnostic criteria. A diagnosis of UTI was made when urine culture was positive with significant bacterial growth $\left(\geq 10^{3}\right.$ colony forming units per $\mathrm{ml}$ ), and no concurrent uroliths were identified. A urolithiasis was diagnosed via abdominal ultrasound or radiographs. Obstructive FLUTD was considered in cats with overdistended, rigid, and painful urinary bladder that was unable to express by gentle palpation. In cats which had obstructive FLUTD caused by urethral plugs detected on radiographs or during catheterization, uroliths or significant bacterial growth had to be eliminated. If no urolith was identified on abdominal ultrasound/radiographs, no urethral plug was observed and urine culture returned negative, the diagnosis of FIC was made.

\section{Urinalysis}

Only cats with urine samples collected by cystocentesis or sterile catheterization were included. Urine specific gravity measurement by refractometer (A. KRÜSS Optronic, GmbH, Hamburg, Germany), urine dipstick analysis (HeptaPhan, Erba Lachema, s.r.o., Brno, Czech Republic), microscopical examination of native urine sediment, and dipslide urine culture were performed in all cats. Dipslide urine bacteriology was performed on cysteine-, lactose-, and electrolyte-deficient and MacConkey medium (Uricult, Orion Diagnostica, Espoo, Finland) according to the manufacturer's instructions. Microbiological culture with sensitivity testing was performed at the Department of Infectious Diseases and Microbiology, UVPS. The samples were plated onto blood agar and MacConkey agar (Oxoid, Hampshire, United Kingdom) and incubated at $37^{\circ} \mathrm{C}$ for $24 \mathrm{~h}$. Isolates were identified using MALDI-TOF Mass Spectrometry (Bruker Daltonic, Germany).

A standard volume of urine $(4 \mathrm{ml})$ was used for urine sediment examination and the urine sediment was examined within $20 \mathrm{~min}$ after collection. After centrifugation of urine at $350 \mathrm{~g}$ for $5 \mathrm{~min}$, the supernatant was removed and the sediment was resuspended in $0.4 \mathrm{ml}$ of supernatant. Haematuria was diagnosed if more than 10 erythrocytes were counted per $\times 400$ field. Pyuria was diagnosed when more than 5 white blood cells (WBC) were recorded per $\times 400$ field in sediment sample. The amount of crystals was rated as one, two, or three crosses. 
Statistical analysis

For all statistical tests and box plot investigation of age and urine specific gravity among groups, a commercial software (Prism, version 7, GraphPad, La Jolla, CA, USA) was used. Descriptive statistics were generated to report continuous data (age, USG; median, minimum, maximum, mean and standard deviation). Based on an assessment of normality (Shapiro-Wilk test) and homoscedasticity (Levene's test), Kruskal-Wallis test followed by Dunn's post hoc test were performed to compare continuous variables between groups. Fisher's exact test was used for the comparison of categorical parameters between groups and odds ratios and $95 \%$ confidence intervals were calculated. Significance was set at $P<0.05$.

\section{Results}

Search of the small animal clinic's records revealed 185 patients with clinical signs consistent with LUTS. Previous catheterization in private practice was performed in 3 cases, 3 cats had undergone perineal urethrostomy, one cat had been treated with glucocorticoids

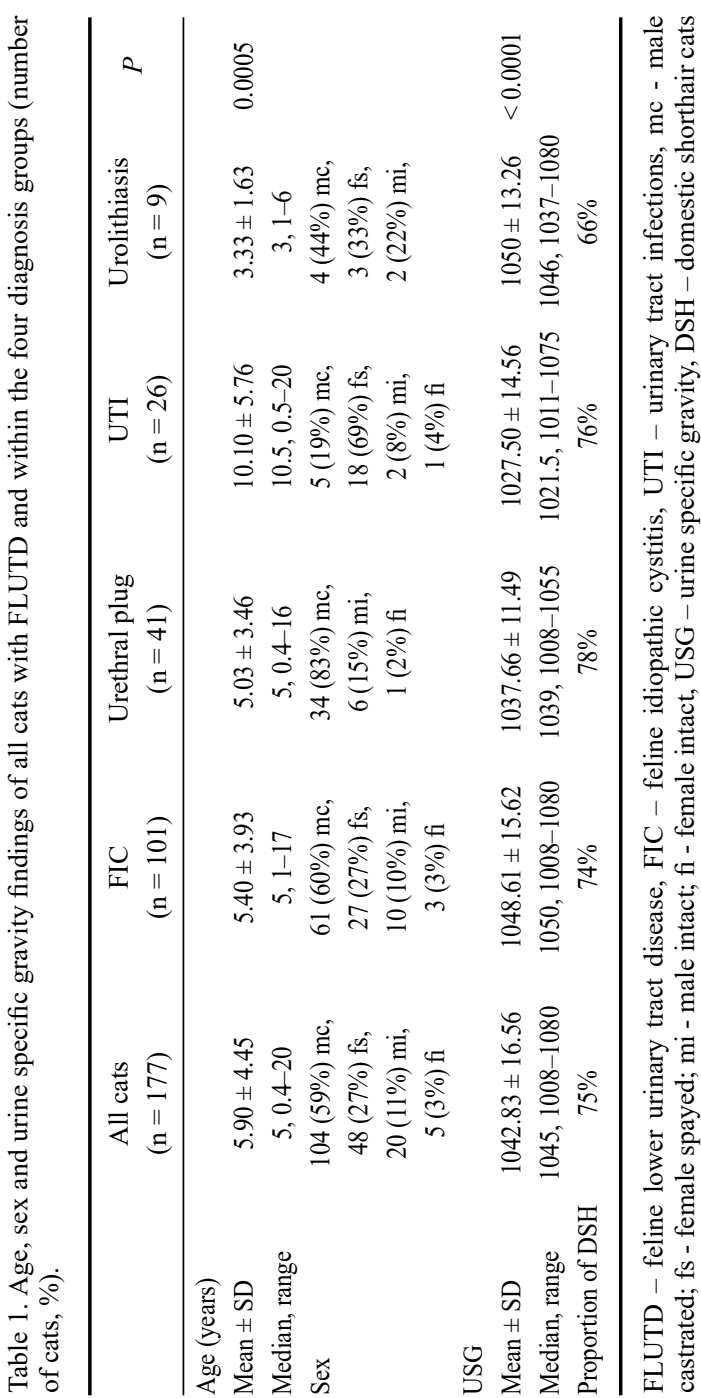
because of atopic dermatitis, and one cat had been treated with antibiotics without a previously performed urine culture. This resulted in a study population of 177 cats.

One hundred and sixty-eight (95\%) of all patients were first opinion cases and eight patients (5\%) were referred from private practices. The studied population consisted of 133 domestic shorthair cats $(75 \%), 23(13 \%)$ British shorthair cats, 5 (3\%) Ragdolls, 4 (2\%) Persian and $4(2 \%)$ Siberian cats and $2(1 \%)$ Russian cats. The other breeds were Ocicat, Norwegian forest cat, Abyssinian cat, Main coon cat, Chartreux cat and Neva masquerade cat each with one representative. Data regarding age and sex are presented in Table 1.

Forty-one $(23 \%)$ cats were diagnosed with a urethral plug, 26 (14\%) with UTI, $9(5 \%)$ patients with urolithiasis and 101 cats $(57 \%)$ with FIC. In 156 (88\%) cases, urine samples were collected via cystocentesis, and $21(12 \%)$ via sterile catheterization.

Cats diagnosed with UTI were significantly older than cats with feline idiopathic cystitis $(P=0.0015)$, urethral plugs $(P=0.0038)$ and urolithiasis $(P=$ $0.0059)$ (Fig. 1). Urinary tract infection was diagnosed significantly more often in patients older than 10 years $(P<0.0001)$, FIC was less frequent in cats older than 10 years, but the difference was not significant $(P$ $=0.1641$ ) (Table 2). Urinary tract infection was more frequently 


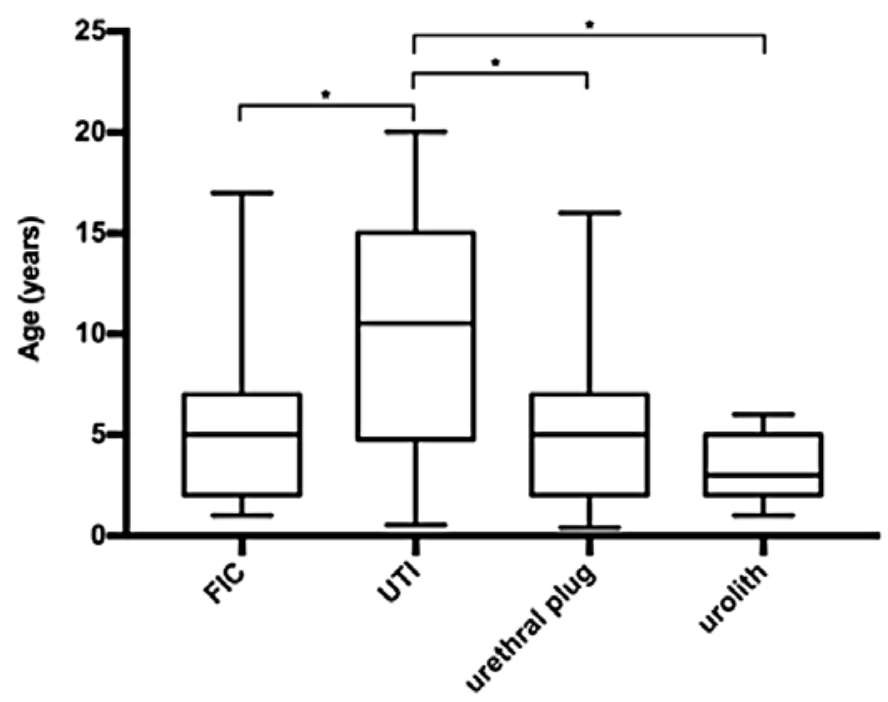

Fig. 1. Data regarding age within groups based on the diagnosis. Significant differences between groups are marked by * $(P=0.0005)$.

FIC - feline idiopathic cystitis, UTI - urinary tract infections

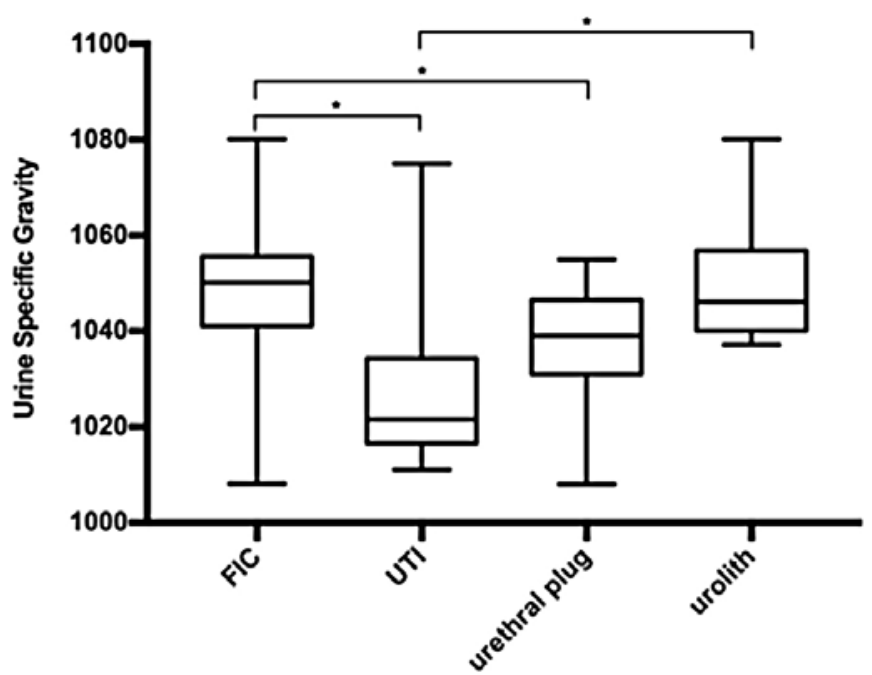

Fig. 2. Urine specific gravity within groups based on the diagnosis. Significant differences between groups are marked by $*(P<0.0001)$

FIC - feline idiopathic cystitis, UTI - urinary tract infections

diagnosed in female cats $(P<0.0001)$ and the diagnosis of urethral plug was made significantly more often in males $(P<0.0001)$ (Table 3$)$. Relationships between the castration status and most frequent diagnoses are presented in Table 4. 
Table 2. Incidence of the four most common causes of FLUTD in cats below 10 years of age and cats aged 10 years or older

\begin{tabular}{lcccc}
\hline Diagnosis & $\begin{array}{c}\text { Cats }<10 \text { years } \\
(\mathrm{n}=146)\end{array}$ & $\begin{array}{c}\text { Cats } \geq 10 \text { years } \\
(\mathrm{n}=31)\end{array}$ & $P$ & Odds ratio \\
\hline FIC & 87 & 14 & 0.1641 & 0.558 \\
Urethral plug & 38 & 3 & 0.0606 & 0.304 \\
UTI & 12 & 14 & $<0.0001$ & 9.196 \\
Urolithiasis & 9 & 0 & & \\
\hline
\end{tabular}

FLUTD - feline lower urinary tract disease, FIC - feline idiopathic cystitis, UTI - urinary tract infections

Table 3. Incidence of the four most common causes of FLUTD in male and female cats.

\begin{tabular}{lcccc}
\hline Diagnosis & Male & Female & $P$ & Odds ratio \\
\hline FIC & 71 & 30 & $>0.9999$ & 1.027 \\
Urethral plug & 40 & 1 & $<0.0001$ & 24.76 \\
UTI & 7 & 19 & $<0.0001$ & 0.107 \\
Urolithiasis & 6 & 3 & $>0.9999$ & 0.848 \\
\hline
\end{tabular}

FLUTD - feline lower urinary tract disease, FIC - feline idiopathic cystitis, UTI - urinary tract infections

Table 4. Incidence of the four most common causes of FLUTD in castrated and intact cats.

\begin{tabular}{lcccc}
\hline Diagnosis & Spayed/castrated & Sexually intact & $P$ & Odds ratio \\
\hline FIC & 88 & 13 & 0.6645 & 1.269 \\
Urethral plug & 34 & 7 & 0.6093 & 0.741 \\
UTI & 23 & 3 & $>0.9999$ & 1.307 \\
Urolithiasis & 7 & 2 & 0.6166 & 0.555 \\
\hline
\end{tabular}

FLUTD - feline lower urinary tract disease, FIC - feline idiopathic cystitis, UTI - urinary tract infections

Table 5. Incidence of pyuria within the diagnosis groups of cats.

\begin{tabular}{lcccc}
\hline Diagnosis & WBC $<5 /$ hpf & WBC $>5 / h p f$ & $P$ & Odds ratio \\
\hline FIC & 92 & 5 & 0.0055 & 0.221 \\
Urethral plug & 35 & 2 & 0.2519 & 0.383 \\
UTI & 13 & 12 & $<0.0001$ & 17.93 \\
Urolithiasis & 9 & 0 & & \\
\hline
\end{tabular}

FIC - feline idiopathic cystitis, UTI - urinary tract infections, WBC - white blood cells, hpf - high power field

The urine specific gravity was significantly lower in cats diagnosed with UTI compared to cats from the FIC group $(P<0.0001)$, and cats with urolithiasis $(P=0.0083)$. Cats from the UTI group had lower USG than cats with urethral plugs, but the difference was not significant $(P=0.1912)$. Data regarding urine specific gravity in different groups are noted in Table 1 and Fig. 2. Cats diagnosed with an infection of Staphylococcus felis had a significantly higher urine specific gravity $(P=0.0137)$ than cats having another isolate identified. Pyuria occurred significantly more often in the UTI group $(P<0.0001)$ than in 
the other studied groups (Table 5). Struvite crystals were identified significantly more often in cats diagnosed with a urethral plug $(P=0.0155)$ (Table 6). Crystals were identified in 15 of 41 cats with the urethral plug, struviteswere identified in all cases,and in two of these, struvites were found together with calcium oxalate crystaluria.

Table 6. Incidence of crystalluria within the diagnosis groups of cats.

\begin{tabular}{lcccc}
\hline Diagnosis & Crystals & No crystals & $P$ & Odds Ratio \\
\hline FIC & 20 & 81 & 0.5815 & 0.796 \\
Urethral plug & 15 & 26 & 0.0155 & 2.834 \\
UTI & 1 & 25 & 0.0181 & 0.123 \\
Urolithiasis & 2 & 7 & $>0.999$ & 1.048 \\
\hline
\end{tabular}

FIC - feline idiopathic cystitis, UTI - urinary tract infections

Of the 26 patients with positive displide urine culture, aerobic bacteriology was performed in 18 patients and infections with single isolate were detected. The identified isolates having significant bacterial growth were Escherichia coli from 9 of the cases, Staphylococcus felis from 4 cases, Staphylococcus intermedius from one patient, Klebsiella spp.from one patient, Enterococcus spp. from one patient, Bacillus spp. from one patient and Achromobacter spp. from one patient. Microbial culture was not performed in 8 patients due to owners' disapproval. Urine culture was positive in $24 / 156$ samples $(15.4 \%)$ from cystocentesis and in $2 / 21$ samples $(9.5 \%)$ collected by sterile catheterization.

\section{Discussion}

In the present study, $75 \%$ of presented cats were domestic shorthair cats, which is in accordance with the population of our patients at UVPS. The majority $(70 \%)$ of patients in our study were male cats, as in previous studies (Gerber et al. 2005; Saevik et al. 2011; Dorsch et al. 2014). One possible explanation is the predisposition of male cats to urinary tract obstruction because of the narrow penile part of the urethra. The urgent nature of urethral obstruction could be the reason why owners contact veterinarian immediately unlike in non-obstructive cases with possible higher rates of spontaneous recovery (Hostutler et al. 2005). Male cats were presented dominantly in all diagnostic groups with the exception of the UTI group, which agrees with the hypothesis that female cats are more prone to UTI because of their shorter urethra (Litster et al. 2009).

Some previous studies indicate that FLUTD occurs more commonly during the winter season (Lekcharoensuk et al. 2001; Eggertsdottir et al. 2007), however, for unknown reason. Cats in our studywere presented more frequently during winter $(30 \%)$ and summer (30\%) seasons than during autumn (21\%) and spring (19\%).

Feline idiopathic cystitis was the most common diagnosis made in $57 \%$ of patients in our study investigating the Czech population of cats with FLUTD. This is in agreement with results of previous studies with an estimated prevalence of FIC at 51 to $63 \%$ (Kruger et al. 1991; Lekcharoensuk et al. 2001; Gerber et al. 2005; Saevik et al. 2011; Dorsch et al. 2014).

The second most common diagnosis in our study were urethral plugs ( $23 \%$ of cases). Clinically, it is difficult to differentiate between obstructive uropathy caused by urethral inflammation, swelling and/or sphincter contraction from a urethral plug (Kruger et al. 2009). Therefore, for a safe identification of a urethral plug, contrast radiography and/or cystoscopy is necessary (Chew et al. 1996). However, these procedures were not routinely performed inthe presentstudy, mainly because of the acute nature of urethral obstruction and the need of prompt intervention. This might have led to misdiagnosis of urethral plugs 
and an underestimation of FIC. The cause of plug formation is still not completely known. Osborne et al. (1992) proposed that crystalluria with concomitant inflammation leads to formation of matrix-crystalline plugs. Interestingly, struvite crystals as the mineral component in urethral plugs still predominate, despite the increase in prevalence of feline calcium oxalate urolithiasis. It is believed that leakage of plasma proteins into urine during the inflammatory process leads to an increase of urinary $\mathrm{pH}$ which promotes further precipitation of struvite crystals. Crystalluria has been shown to occur more frequently in cats with FIC than in cats with other forms of FLUTD (Kruger et al. 1991). In our study, only cats from the urethral plug group had struvites identified more frequently on urinalysis than cats with other diagnoses. Higher incidence of crystalluria in cats with plugs confirms that crystalluria is one of the important predisposition factors in obstructive FLUTD.

The most noticeable disagreement was found in the prevalence of UTI in the population of Czech cats. The previously reported incidence rate of UTI in the USA was determined to be below 3\% (Kruger et al. 1991; Buffington et al. 1997). European researchers determined higher prevalence of UTI in cats. Incidence rate of $22.2 \%$ was described by Kraijer et al. (2003), $8 \%$ by Gerber et al. (2005), $11.8 \%$ by Saevik et al. (2011) and $18.9 \%$ by Dorsch et al. (2014). In our study, $14 \%$ of patients had primary UTI, which is in agreement with other European researchers. Studies in the USA were performed at referral institutions and included mainly referred cases; in contrast, most European studies comprised predominantly first-opinion cases with only a minor portion of referred patients, which makes the main difference in the studied population. The mean age of all patients in our study was 5.9 years, which was similar to studies from the USA (5.1 years) (Buffington et al. 1997) and Norway (5.6 years) (Saevik et al. 2011). The incidence of UTI in cats $<10$ years in our study was $8.2 \%$. This finding supports the clinical experience that infections should be considered as a possible cause of FLUTD not only in older but even in young and middle-aged cats with LUTS.

Collecting the urine sample by cystocentesis is the gold standard for bacteriological testing (Pressler and Bartges 2009). Twenty-one urine samples were collected by sterile catheterization in our study, to prevent possible complications after performed cystocentesis in obstructed patients with overdistended bladder. Cystocentesis was performed in $92.3 \%$ $(24 / 26)$ of cases in cats diagnosed with primary UTI.

The distribution of bacterial species is in accordance with previously published reports, with Escherichia coli being the most commonly found microorganism (Wooley and Blue 1976; Litster et al. 2007; Passmore et al. 2008). It was identified in 50\% of our patients, and the second most common isolate identified in $22 \%$ of cases was Staphyloccocus felis. Staphylococcal species are commonly urease producing organisms.Urease alkalizes urine and, especially in combination with higher urine specific gravity, leads to the formation of struvite crystals (Litster et al. 2007). Infection of Staphyloccocus felis was associated with higher urine specific gravity in our study, as previously noted by Litster et al. (2007), and resulted in crystalluria in $25 \%$ of patients, but did not result in urolithiasis in any of our patients. Two of the isolates identified (Achromobacter spp., Bacillus spp.) are not common primary urinary tract pathogens. Urinary tract infections caused by Achromobacter spp. and Bacillus spp. are rarely noted in humans. Both of these isolates are recognized as opportunistic pathogens that can cause systemic or local infections including urinary tract infections in immunocompromised patients and patients with urological or other underlying diseases in humans (Tena et al. 2008; Pérez Barragán et al. 2018; Ehling-Schulz et al. 2019). Both feline patients suffered from a concurrent chronic kidney disease. Their urine samples were collected by cystocentesis, but we cannot rule out with certainty the possibility of contamination during the handling and processing of the urine sample.

The results of our study confirm previous findings of the American and European studies regarding FIC being the most frequent cause of LUTS in feline patients. The prevalence 
of UTI in our study was higher than in the American patients, but in agreement with the European population of cats. Even though the risk of UTI is higher in females and increases with age, UTI should always be considered as a possible cause and eliminated during the diagnostic work-up even in younger and middle-aged cats presented with LUTS.

\section{Conflict of Interest}

The authors disclose no conflict of interest. None of the authors have any financial or personal relationships that could inappropriately influence or bias the content of the paper.

\section{Off-label Antimicrobial Declaration}

The authors declare that there was no off-label use of antimicrobials.

\section{References}

Bailiff NL, Nelson RW, Feldman EC, Westropp JL, Ling GV, Jang SS, Kass PH 2006: Frequency and risk factors for urinary tract infection in cats with diabetes mellitus. J Vet Intern Med 20: 850-855

Bailiff NL, Westropp JL, Nelson RW, Sykes JE, Owens SD, Kass PH 2008: Evaluation of urine specific gravity and urine sediment as risk factors for urinary tract infections in cats. Vet Clin Pathol 37: 317-322

Buffington CA, Chew DJ, Kendall MS, Scrivani PV, Thompson SB, Blaisdell JL, Woodworth BE 1997: Clinical evaluation of cats with nonobstructive urinary tract diseases. J Am Vet Med Assoc 210: 46-50

Chew DJ, Buffington T, Kendall MS, Osborn SD, Woodsworth BE 1996: Urethroscopy, cystoscopy, and biopsy of the feline lower urinary tract. Vet Clin North Am Small Anim Pract 26: 441-462

Dorsch R, Remer C, Sauter-Louis C, Hartmann K 2014: Feline lower urinary tract disease in a German cat population. A retrospective analysis of demographic data, causes and clinical signs. Tierarztl Prax Ausg K Kleintiere Heimtiere 42: 231-239

Eggertsdottir AV, Lund HS, Krontveit R, Sorum H 2007: Bacteriuria in cats with feline lower urinary tract disease: a clinical study of 134 cases in Norway. J Feline Med Surg 9: 458-465

Ehling-Schulz M, Lereclus D, Koehler TM 2019: The Bacillus cereus group: Bacillus species with pathogenic potential. Microbiol Spectr 7 (3)

Forrester SD, Towell TL 2015: Feline idiopathic cystitis. Vet Clin North Am Small Anim Pract 45: 783-806

George EL 1996: Bacterial urinary tract infections. Vet Clin North Am Small Anim Pract 26: 297-304

Gerber B, Boretti FS, Kley S, Laluha P, Muller C, Sieber N, Unterer S, Wenger M, Fluckiger M, Glaus T, Reusch CE 2005: Evaluation of clinical signs and causes of lower urinary tract disease in European cats. J Small Anim Pract 46: $571-577$

Gunn-Moore D 2003: Feline lower urinary tract disease. J Feline Med Surg 5: 133-138

Hostutler RA, Chew DJ, DiBartola SP 2005: Recent concepts in feline lower urinary tract disease. Vet Clin North Am Small Anim Pract 35: 147-170

Kraijer M, Fink-Gremmels J, Nickel RF 2003: The short-term clinical efficacy of amitriptyline in the management of idiopathic feline lower urinary tract disease: a controlled clinical study. J Feline Med Surg 5: 191-196

Kruger JM, Osborne CA, Goyal SM, Wickstrom SL, Johnston GR, Fletcher TF, Brown PA 1991: Clinical evaluation of cats with lower urinary tract disease. J Am Vet Med Assoc 199: 211-216

Kruger JM, Osborne CA, Lulich JP 2009: Changing paradigms of feline idiopathic cystitis. Vet Clin North Am Small Anim Pract 39: 15-40

Lekcharoensuk C, Osborne CA, Lulich JP 2001: Epidemiologic study of risk factors for lower urinary tract diseases in cats. J Am Vet Med Assoc 218: 1429-1435

Lew-Kojrys S, Mikulska-Skupien E, Snarska A, Krystkiewicz W, Pomianowski A 2017: Evaluation of clinical signs and causes of lower urinary tract disease in Polish cats. Vet Med (Praha) 62: 386-393

Litster A, Moss SM, Honnery M, Rees B, Trott DJ 2007: Prevalence of bacterial species in cats with clinical signs of lower urinary tract disease: recognition of Staphylococcus felis as a possible feline urinary tract pathogen. Vet Microbiol 121: 182-188

Litster A, Moss S, Platell J, Trott DJ 2009: Occult bacterial lower urinary tract infections in cats-urinalysis and culture findings. Vet Microbiol 136: 130-134

Mayer-Roenne B, Goldstein RE, Erb HN 2007: Urinary tract infections in cats with hyperthyroidism, diabetes mellitus and chronic kidney disease. J Feline Med Surg 9: 124-132

Osborne CA, Kruger JM, Lulich JP 1996: Feline lower urinary tract disorders. Definition of terms and concepts. Vet Clin North Am Small Anim Pract 26: 169-179

Osborne CA, Kruger JP, Lulich JP, Bartges JW, Polzin DJ, Molitor T, Beauclair KD, Onffroy J 1992: Feline matrix-crystalline urethral plugs: A unifying hypothesis of causes. J Small Anim Pract 33: 172-177

Passmore CA, Sherington J, Stegemann MR 2008: Efficacy and safety of cefovecin for the treatment of urinary tract infections in cats. J Small Anim Pract 49: 295-301

Pérez Barragán E, Sandino Pérez J, Corbella L, Orellana MA, Fernández-Ruiz M 2018: Achromobacter xylosoxidans bacteremia: clinical and microbiological features in a 10-year case series. Rev Esp Quimioter 31: $268-273$ 
Pressler B, Bartges JW 2009: Chapter 313 - Urinary tract infections. In: Ettinger SJ, Feldman EC (Eds): Textbook of Veterinary Internal Medicine Expert Consult $7^{\text {th }}$ Edition. Saunders, Edinburgh, pp. 2026-2047

Saevik BK, Trangerud C, Ottesen N, Sorum H, Eggertsdottir AV 2011: Causes of lower urinary tract disease in Norwegian cats. J Feline Med Surg 13: 410-417

Tena D, Gonzalez-Praetorius A, Perez-Balsalobre M, Sancho O, Bisquert J 2008: Urinary tract infection due to Achromobacter xylosoxidans: report of 9 cases. Scand J Infect Dis 40: 84-87

Wooley RE, Blue JL 1976: Quantitative and bacteriological studies of urine specimens from canine and feline urinary tract infections. J Clin Microbiol 4: 326-329 